\title{
Lineamientos de política pública para buenas prácticas en uso educativo de las TIC: Una propuesta desde el Caribe Colombiano Public policy guidelines for good practices in educational use of ICT: A proposal from the Colombian Caribbean
}

\author{
Alba Zulay Cárdenas Escobar ${ }^{1}$, Alix Pacheco Turizo ${ }^{2}$ \\ acardenas@unitecnologica.edu.co,apachecot@unicartagena.edu.co \\ ${ }^{1}$ Facultad de Economía y Negocios \\ Universidad Tecnológica de Bolívar \\ Cartagena, Colombia \\ ${ }^{2}$ Facultad de Educación \\ Universidad de Cartagena \\ Cartagena, Colombia
}

\begin{abstract}
Resumen- Este documento busca trascender los resultados obtenidos en el Programa de Investigación "Construcción de buenas prácticas en uso educativo de TIC en las escuelas innovadoras del Caribe Colombiano", ejecutado por el Centro de Innovación Educativa Regional CIER Norte, entre los años 2014 a 2016, al proponer lineamientos de política pública para el desarrollo de buenas prácticas en uso de TIC, más allá del contexto regional y nacional, en tópicos como: dotación de infraestructura tecnológica, desarrollo de contenidos educativos digitales, diseño de estrategias pedagógicas, formación de docentes y el desarrollo de competencias básicas y ciudadanas en estudiantes en las escuelas oficiales.
\end{abstract}

Palabras clave: Lineamientos de Política Pública, Centros de Innovación Educativa Regionales, Escuelas Innovadoras, Formación Docente, Contenidos Digitales, Competencias.

Abstract- This document seeks to transcend the results obtained in the Research Program "Building Good Practices in ICT Educational Use in Innovative Schools in the Colombian Caribbean," implemented by RIC North Regional Educational Innovation Center between 2014 and 2016, to propose public policy guidelines for the development of good practices in ICT use, beyond the regional and national context, in topics such as: provision of technological infrastructure, development of digital educational content, design of pedagogical strategies, teacher training and development of basic and citizens competences in students in the official schools.

Keywords: Guidelines for Public Policy, Regional Educational Innovation Centers, Innovative Schools, Teacher Training, Digital Content, Competencies.

\section{INTRODUCCIÓN}

En la investigación llevada a cabo se parte de la postura teórica sobre política pública, que plantea Thomas Dye (1995), que la define como "un instrumento para la conducción de sociedades, a través de la cual, los gobiernos definen sus objetivos y asignan los recursos necesarios para garantizar los derechos y atender las necesidades de sus pobladores”.

Partiendo de esta definición, se fijó como objetivo central del programa de investigación "Construcción de buenas prácticas en uso educativo de TIC en las escuelas innovadoras del Caribe Colombiano” del Centro de Innovación Educativa Regional CIER Norte, diseñar lineamientos de política pública que partieran de las realidades constitutivas de las instituciones educativas públicas objeto de análisis, así como del conocimiento del uso y de la gestión que se le ha dado a las TIC en los procesos adelantados por dichas comunidades educativas, lo cual implicó un ejercicio de análisis crítico para propiciar el desarrollo de una cultura digital con pertinencia social, que no quede en el cumplimiento de indicadores y metas, sino que efectivamente impacte el contexto de las instituciones educativas.

A partir de la realidad encontrada, las "Escuelas Innovadoras” denominadas así en el convenio marco del programa de investigación del Centro de Innovación Educativa Regional CIER Norte, fueron definidas como aquellas potencialmente dispuestas a: emprender una gestión educativa, orientada a diseñar e implementar estrategias pedagógicas y contenidos educativos digitales en sus prácticas pedagógicas mediadas por TIC; a promover y propiciar la formación de sus docentes en uso de TIC; y, desde allí, apostarle al desarrollo de competencias básicas y ciudadanas de sus estudiantes (Pacheco, Pérez, González, Pombo, Mestre, Román,... \& Henríquez, 2016, p 4, 217). Esta definición se constituyó en fundamento para la formulación de los propósitos investigativos del programa de investigación, al tiempo que perfiló la definición de las categorías nucleares objeto de investigación.

De otra parte, el concepto de buenas prácticas educativas en uso de TIC, se traduce en una gestión educativa contextualizada y mediada por TIC; el diseño, desarrollo y evaluación de estrategias pedagógicas con uso de TIC; el diseño, adaptación y uso de contenidos educativos digitales; la formación continua de docentes en uso educativo de TIC y la investigación sobre la incidencia de su uso en el desarrollo de competencias básicas y ciudadanas de los estudiantes (Pacheco et al., 2016, p 4-6), sobre las cuales debe existir una política pública que trascienda periodos presidenciales, es decir sea sostenible y medible a largo plazo. 


\section{CONTEXTO}

La educación en las dos últimas décadas ha estado influida por tendencias pedagógicas que requieren la modificación de prácticas en la enseñanza. Esto, acompañado del uso de las Tecnologías de la Información y Comunicación (TIC) en la enseñanza, lo cual enriquece el aprendizaje en aspectos como la introducción de la comunicación, la reflexión, el cuestionamiento $\mathrm{y}$ el análisis, forzando a docentes $\mathrm{y}$ estudiantes a repensar y reorientar la forma en que se enseña y se aprende (Gewerc \& Montero, 2011).

\section{A. Antecedentes recientes del uso de TIC en educación.}

En América Latina, según un estudio en el contexto español (Vaillant y Marcelo, 2012), citado por UNICEF en el estudio realizado por Vaillant, (2013) el 28,5\% de los docentes usan las TIC y el $30 \%$ hace un uso ocasional (menos de una vez al mes). El 41,5\% restante de los profesores manifiesta que hace un uso regular y sistemático de las TIC en sus aulas, aunque con grados de intensidad muy diferentes. Cuando los docentes hacen uso de las tecnologías en su enseñanza, lo hacen para transmitir contenidos como apoyo a la exposición oral (78,7\%), para presentar contenido mediante un sistema multimedia o hipermedia (62,3\%), y para realizar demostraciones que permitan simular determinados escenarios (44,5\%). Los docentes no utilizan las tecnologías por varias razones, entre ellas, por la falta de acceso a las computadoras, la carencia de las competencias necesarias, la escasa utilidad para su asignatura y el poco impacto para su centro educativo. Anota además el estudio de la UNICEF que a pesar de que el acceso a dispositivos digitales ha venido aumentado progresivamente en América Latina, muchos de los docentes de la región todavía carecen de acceso a tecnologías y conocimientos básicos sobre ellas.

Según la publicación sobre el uso de las TIC para el aprendizaje y la innovación en los centros escolares de Europa de la Agencia Ejecutiva en el ámbito Educativo, Audiovisual y Cultural (EACE, 2011), la Unión Europea como elemento común establece que todos los países europeos diseñen estrategias para fomentar el uso de las TIC en educación. Continúa el estudio señalando que en el 2010, la Comisión Europea adoptó una nueva Agenda Digital para Europa (Comisión Europea, 2011) que reafirma y valora una serie de desafíos clave para los próximos años dentro de los cuales se contempla proporcionar a la población europea un alto grado de competencias TIC a nivel de usuario. Así mismo anota, que 28 países han puesto en marcha una estrategia para las TIC en el ámbito educativo y en su mayoría las estrategias tienen como fin proporcionar a los alumnos las destrezas TIC, así como formación especializada en las TIC al profesorado en su mayoría de educación primaria y secundaria. Las áreas estratégicas más importantes son el E-learning, el desarrollo de competencias digitales en medios de comunicación, la utilización de las TIC en los centros escolares y la inclusión digital.

No obstante, los registros de inserción de TIC en procesos de formación, anotados anteriormente, la UNICEF en el estudio Integración de TIC en los sistemasisepide formación docente inicial y continua para la Educación Básica en América Latina (UNICEF, 2013), afirma que en materia de acceso e infraestructura TIC en las escuelas no aseguran su uso efectivo; por lo tanto, se requiere una fuerte apuesta para preparar a los docentes para que usen de forma innovadora estas tecnologías en sus clases. Para ello, hay dos opciones básicas: la formación continua de los docentes en servicio que responde a demandas coyunturales y su integración a la formación inicial docente.

Por otra parte, según el informe de Internet Society (2015) de los proyectos de investigación o estudios realizados en el campo de las TIC, también han resultado instrumentos fundamentales para la implantación de sus estrategias generales sobre las TIC. Dichos proyectos mejoran la comprensión sobre los efectos de su uso y, por tanto, contribuyen a aumentar la eficacia de las estrategias que se desarrollan, lo cual resulta especialmente importante en los centros escolares, pues se ha identificado que es en este nivel, en el que se han puesto en marcha más proyectos de investigación en todos los países.

De acuerdo con la UNESCO (2013), la actualización educativa implica, en primer lugar un desafío pedagógico, para incorporar las TIC al aula y en el currículum escolar, la adecuación de la formación inicial y en servicio de los docentes, y políticas públicas que aseguren la implementación sistémica de reformas que impacten en los sistemas educativos de manera integral, lo que incluye asegurar la cobertura y calidad de la infraestructura tecnológica (hardware, software y acceso a servicios de información y comunicación). Junto con esto, las TIC también presentan potenciales beneficios para mejorar la gestión escolar, lo que implica además preparar a directivos y administrativos en estas nuevas tecnologías. Las TIC [...] no son la solución, ni la panacea frente a las dificultades que enfrenta la comunidad educativa, pero sí pueden ser un valioso recurso que los docentes desde sus perspectivas y necesidades particulares deben explorar y utilizar convenientemente para favorecer la dinámica escolar [...] (Mestre G, Díaz D., 2012, p. 80)

B. Origen de los Centros de Innovación Educativa Regionales en Colombia: Trazabilidad y Propósitos.

Los Centros de Innovación Educativa Regionales tienen su origen en el documento CONPES 3507, que autorizó al gobierno nacional de Colombia a contratar un crédito con la banca internacional para financiar el macroproyecto "ICT Capability Bulding - Construyendo capacidad en el uso de las TIC para innovar en la educación”, siendo el objetivo de este crédito "consolidar la política de mejoramiento de la calidad de la educación mediante la conformación de espacios de interacción e intercambio que fomenten la construcción de conocimiento y el desarrollo de competencias" ( Departamento Nacional de Planeación DNP, 2008, p 11). En ese documento se definió como una de las estrategias la conformación de cinco (5) Centros de Innovación Educativa Regionales, a partir de convocatorias en las regiones del país para la conformación de alianzas interinstitucionales públicoprivadas.

Los cinco (5) Centros de Innovación Educativa Regionales, tendrían por finalidad cerrar las brechas inter e intrarregionales en uso de TIC para innovar en educación mediante: la producción de más de 33 mil contenidos educativos digitales de alta calidad, en las áreas de matemáticas, ciencias Naturales y lenguaje para la educación preescolar, básica y media; la formación de más de 16 mil docentes colombianos en el uso pedagógico de TIC y el desarrollo de contenidos educativos digitales que contribuirían al enriquecimiento y actualización del Portal Educativo Colombia Aprende. Todo ello, soportado 
en la dotación de infraestructura tecnológica a los Centros de Innovación y a las instituciones educativas oficiales denominadas en el marco del proyecto, como "Escuelas Innovadoras".

Así mismo, el CONPES 3582 estableció la "Política Nacional de Ciencia, Tecnología e Innovación”, destacando entre sus estrategias el fomento y reconocimiento de centros de investigación y desarrollo tecnológico del Sistema Nacional de Ciencia, Tecnología e Innovación - SCTeI y fijando como lineamientos de acción el fortalecimiento de las capacidades regionales para la generación, gestión y uso del conocimiento (DNP, 2009, 33-34, 52). Coherente con esta directriz, se planteó la estrategia de impulsar la investigación en instituciones de educación básica, media y superior (DNP, 2009, p 53). De allí que las más recientes convocatorias de Colciencias (entidad estatal que lidera, orienta y coordina la política nacional de SCTeI en Colombia), las No 716 y 765 del 2015, en sus términos de referencia, apuntaron a atender en primera instancia, las necesidades de los Centros de Innovación Educativa Regional y en segunda instancia, la postulación a la conversión de dichos Centros de Innovación en Centros del SNCTeI en educación.

Con fundamento en las directrices emanadas de los CONPES, y alineado con lo dispuesto en el Plan Decenal de Educación 2006-2016 y el Plan de Desarrollo Nacional “Camino a la Prosperidad” 2010 - 2014, el Ministerio de Educación Nacional MEN de Colombia formula el proyecto "ICT Education Capability Building - Construyendo capacidades en Uso Educativo de las TIC", y conjuntamente con el Fondo de Cooperación para el Desarrollo Económico de Corea del Sur, a finales del año 2011, siguiendo lo dispuesto en el CONPES 3507 (DNP, 2008, p 1), a través de convocatoria pública, se selecciona como consultor coreano la firma Korea Education \& Research Information ServiceKERIS, que asumiría la responsabilidad del diseño del proyecto y la estructuración del proceso de selección de la firma implementadora y se condiciona la firma del contrato, al conocimiento previo de los sitios donde se implementaría el proyecto, y a la conformación de las alianzas regionales que darían vida a cada Centro de Innovación Educativa Regional.

El 16 de septiembre de 2013, el proyecto "ICT Capability Building” y sus Centros de Innovación Educativa Regionales son declarados en el CONPES 3768 prioridad de estado y vehículo para hacer de Colombia el país de Latinoamérica bandera en producción de contenidos educativos digitales, formación docente y proyectos de investigación (DNP, 2013, p 7, 11). Así mismo, mediante convocatoria internacional, en octubre de 2013, se selecciona a la compañía LG CNS Ltda., empresa de Corea del Sur, como implementadora del proyecto, en razón de sus logros en el uso de tecnologías de la información.

En este contexto, la Universidad Tecnológica de Bolívar UTB, es seleccionada en julio de 2013 como universidad operadora del Centro de Innovación Educativa Regional CIER Norte y se protocoliza la "Alianza CIER Norte", alianza de naturaleza público-privada, ubicada en la ciudad de Cartagena de Indias, Colombia, con la participación seis (6) secretarias de educación territoriales y seis (6) instituciones de educación superior públicas y privadas.

El 24 de enero de 2014 se firma el Convenio de Asociación 0285 entre el MEN y la UTB, como IES operadora de la
Alianza. La duración del convenio se fija en 30 meses y se establecen las siguientes metas: $1^{\mathrm{a}}$ ) formación de mínimo tres mil (3.000) docentes de la región Caribe Colombiana de los niveles de básica y media, de instituciones educativas oficiales; y $2^{\mathrm{a}}$ ) la producción de mínimo seis mil (6.000) contenidos educativos digitales para el aprendizaje, empaquetados en seis (6) planes de estudio ( coursewares, en inglés) de las áreas de lenguaje, matemáticas y ciencias naturales para $6^{\circ}$ y $7^{\circ}$ grado, diseñados en correspondencia con los estándares curriculares y derechos básicos de aprendizaje - DBA, definidos por el MEN, con sus respectivos metadatos y publicados en el Portal Colombia Aprende, el repositorio de contenidos educativos digitales oficial gratuito más grande del país.

En concordancia con las acciones específicas que desde su concepción se fijaron a los Centros de Innovación Educativa Regionales, el MEN mediante convocatoria pública, selecciona en el año 2013 cincuenta (50) instituciones educativas llamadas en el marco del convenio "Escuelas Innovadoras" localizadas en las distintas regiones del país, diez en cada región de influencia de los Centros de Innovación Educativa Regionales.

Para la región Caribe Colombiana, se seleccionaron diez (10) instituciones educativas oficiales: cinco (5) de ellas en el departamento de Bolívar, dos (2) en Sincelejo, dos (2) en Atlántico y una ( 1) en San Andrés Islas.

En el mismo año 2013, Colciencias y el MEN, también por convocatoria, impulsaron a través de las diferentes alianzas interinstitucionales que dieron vida a los Centros de Innovación Educativa Regionales, la presentación de propuestas de programas de investigación enfocadas en innovación educativa con uso de las TIC, a desarrollarse de manera conjunta en y con los miembros de la comunidad educativa de las "Escuelas Innovadoras".

En el Caribe Colombiano, la Alianza CIER Norte, bajo la operación y representación de la UTB, presentó el programa de investigación "Construyendo buenas prácticas en uso educativo de TIC, en las Escuelas Innovadoras del Caribe Colombiano, que impacten la calidad educativa en la región”, bajo el liderazgo de los grupos de investigación de las IES parte de la Alianza: "Educación, Universidad y Sociedad” de la Universidad de Cartagena UdeC; "Educación e Innovación Educativa” de la UTB; Kuagro, de la Fundación Universitaria Colombo Americano y GISEDH, de la Institución Tecnológica ITSA

En Julio de 2014 se firma el convenio 048 de 2014 entre Colciencias y la UTB, de duración 15 meses, a través del cual Colciencias se compromete a cofinanciar el programa de investigación y las instituciones de educación superior participantes miembros de la Alianza CIER Norte a desarrollar el programa de investigación en las diez (10) "Escuelas Innovadoras” seleccionadas en la región Caribe Colombiana.

\section{DESCRIPCIÓN}

La investigación se diseñó metodológicamente, con fundamento en el paradigma socio-crítico, desde el cual se pretende interpretar y comprender las realidades, que en torno a las TIC, se construyen en las "Escuelas Innovadoras"; promover el empoderamiento de los diferentes actores de las comunidades educativas sobre las problemáticas detectadas en 
la implementación de las TIC, como mediadoras de la gestión educativa; propiciar la construcción colectiva de alternativas de solución a las problemáticas identificadas y tomar decisiones en torno a su solución.

Coherentes con el paradigma asumido, la investigación se abordó desde un enfoque cualitativo, en cuya implementación se convocó la selección de técnicas de investigación como la aplicación de cuestionarios, el desarrollo de grupos focales, la revisión documental, la entrevista, los conversatorios y la sistematización de experiencias.

\section{Resultados}

Transcurridos los 30 meses del convenio que dio vida y operatividad al Centro de Innovación Educativa Regional Norte, se evidencia un fortalecimiento de capacidades y competencias en el uso de las TIC al servicio de la innovación en la educación en el Caribe Colombiano a través de los ejes de intervención que se describen en los siguientes apartados.

\section{A. Formación Docente.}

La formación de 4.509 docentes de escuelas oficiales e IES a través de los programas: CREATIC Inspirar, Crear $y$ Diseñar con TIC, principalmente; Colegios 10TIC y Aulas Fundación Telefónica. El impacto en los diferentes departamentos de la Región Caribe adicionando los datos del departamento de Quindío, intervenido en alianza con otro Centro de Innovación Educativa Regional, se pueden ver en la Fig. 1.

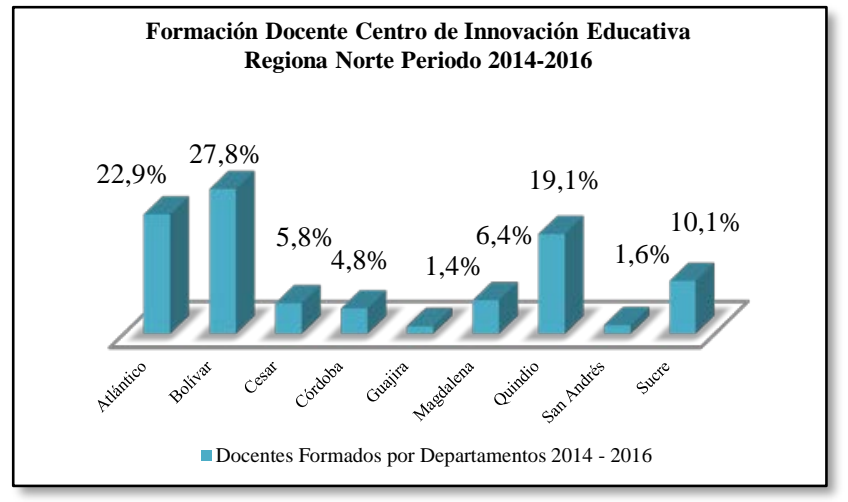

Figura 1 Formación docente periodo 2014 - 2016. Fuente: Archivos del Centro de Innovación Educativa Regional Norte (2016)

\section{B. Diseño y desarrollo de contenidos educativos digitales.}

El diseño y desarrollo de más de 13.330 (de un global de más de 50.000 a nivel nacional) contenidos educativos digitales, empaquetados en 196 objetos de aprendizaje, 33 unidades didácticas y 6 planes de estudio por niveles o coursewares, todos con sus respectivos metadatos y publicados y disponibles de manera gratuita en el Portal Colombia Aprende del Ministerio del MEN, en el enlace http://aprende.colombiaaprende.edu.co/sites/default/files/nasp ublic/ContenidosAprender/index.html

\section{Resultados del programa investigación}

El programa de investigación del Centro de Innovación Educativa Regional Norte, en primera instancia, bajo el liderazgo del grupo de investigación Kuagro, se propuso, reconocer los lineamientos de buenas prácticas en uso educativo de TIC en la gestión educativa de las Escuelas Innovadoras del Caribe Colombiano. Para ello, hizo la caracterización del contexto sociodemográfico del área de influencia de las Escuelas Innovadoras; el reconocimiento de las expectativas, necesidades e intereses a los que debe responder una gestión educativa en uso de TIC, con pertinencia social; así mismo, se caracterizaron los procesos de inclusión de TIC en la gestión directiva, administrativa, académico-curricular y de gestión de la comunidad, con miras a la identificación de factores que favorecen y limitan el uso de TIC. Con fundamento en la anterior caracterización, se identificaron y sistematizaron las buenas prácticas educativas en uso de TIC en la gestión educativa a partir de la dotación de infraestructura tecnológica y los procesos de formación docente desarrollados desde el Centro de Innovación Educativa Regional Norte.

En segunda instancia, liderado por el Grupo de Investigación GISEDH, el programa de investigación, se planteó el propósito de evaluar la pertinencia del uso de estrategias pedagógicas mediadas por TIC, en el desarrollo de competencias básicas y ciudadanas en los estudiantes de los grados $6^{\circ}$ y $7^{\circ}$ de las instituciones educativas intervenidas. Para el logro del propósito planteado se hizo primero el reconocimiento de estrategias pedagógicas exitosas en el contexto nacional e internacional, que pudieran constituirse en referentes de buenas prácticas para el desarrollo de competencias básicas y ciudadanas. Así mismo, se caracterizaron las principales estrategias pedagógicas mediadas por TIC y se analizó su aporte al desarrollo de competencias básicas y ciudadanas. Con fundamento en los resultados se construyó un banco de estrategias pedagógicas mediadas por TIC orientadas al desarrollo de competencias básicas y ciudadanas, producidas por docentes de las Escuelas Innovadoras y una cartilla electrónica (González, G. et al, 2016).

Continuando con el desarrollo de la concepción de Escuelas Innovadoras, en tercera instancia, el programa de investigación, desde el grupo de investigación Educación, Universidad y Sociedad, lideró el propósito de evaluar el aporte de los contenidos educativos digitales al desarrollo de competencias básicas y ciudadanas en los estudiantes de los grados $6^{\circ}$ y $7^{\circ}$ de las Escuelas Innovadoras del Caribe Colombiano. En este sentido, se desarrollaron acciones investigativas encaminadas a la caracterización de los contenidos educativos digitales disponibles en las Escuelas Innovadoras y sus usos en la enseñanza y el aprendizaje de las áreas de lenguaje, matemáticas, ciencias naturales y ciencias sociales. De igual forma, se analizó la experiencia de los estudiantes en uso de contenidos educativos digitales frente al desarrollo de sus estilos de aprendizaje y estilos cognitivos. Como resultado de las anteriores acciones investigativas, se elaboró una cartilla electrónica con la propuesta de lineamientos y pautas para el diseño, producción, gestión y uso, de contenidos educativos digitales que contribuyan al desarrollo de competencias básicas y ciudadanas de los estudiantes de $6^{\circ}$ y $7^{\circ}$ de básica secundaria, (Pombo, A. et al, 2016).

El proyecto de investigación, liderado por el grupo Educación e Innovación Educativa, reconociendo la importancia que tiene la formación docente para el desarrollo de procesos educativos innovadores, se planteó como cuarto propósito investigativo, sistematizar los procesos de formación 
docente en uso educativo de TIC, con miras al reconocimiento de su aporte al desarrollo de las competencias profesionales del docente identificadas por el MEN, en los docentes de las Escuelas Innovadoras del Caribe Colombiano. Para ello, definió como punto de partida de la investigación, el reconocimiento en el contexto nacional e internacional, de buenas prácticas de formación docente en uso de TIC. Una vez analizados estos referentes, bajo un enfoque de sistematización, se procedió a identificar los procesos de formación con uso de TIC en los que han participado los docentes de las Escuelas Innovadoras, como se observa en la Fig. 2 y su incidencia en la práctica pedagógica, así como a la identificación de las competencias desarrolladas en los docentes a partir de los procesos de formación con uso de TIC.

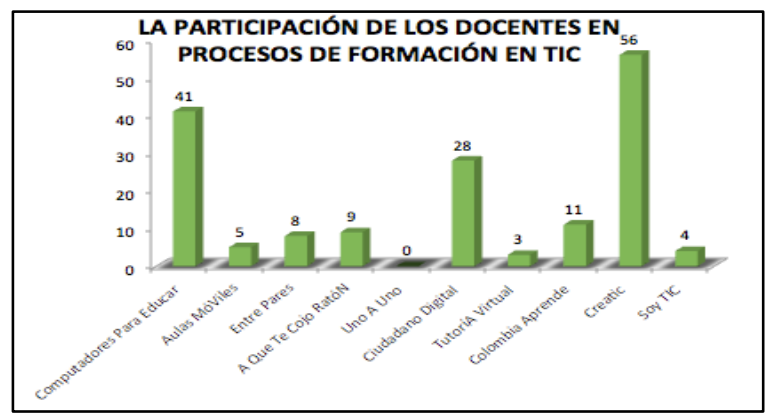

Figura 2 Participación de los Docentes de las Escuelas Innovadoras en procesos de formación en Uso de TIC. Copyright 2016 por Mestre, Cárdenas, Rojas \& Ruiz. Reimpresión con permiso.

Con fundamento en los anteriores resultados, se construyeron lineamientos de buenas prácticas para la formación docente con uso de TIC, que constituyen la base para la formulación de lineamientos de política pública en educación en este tópico y se propuso un nuevo modelo de formación docente que se ilustra en la Fig. 3

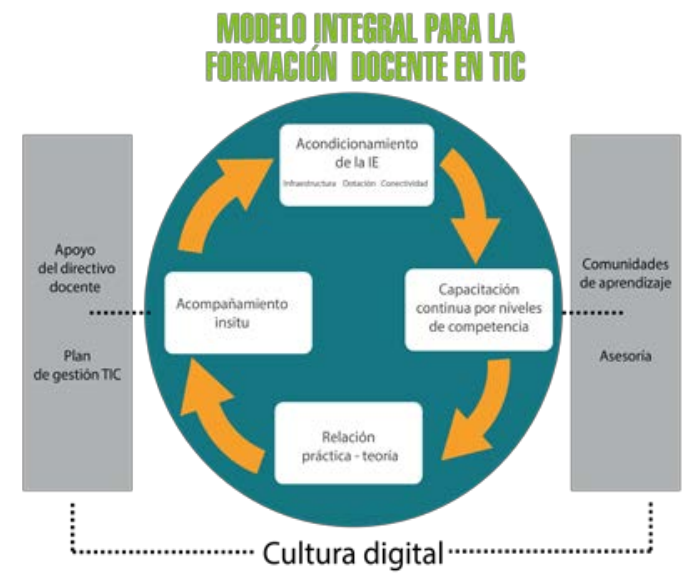

Figura 3 Modelo de Formación Docente en Uso de TIC. Copyright 2016 por Mestre et al. Reimpresión con permiso.

Seguidamente, ante la necesidad de revisar el impacto educativo generado en las Escuelas Innovadoras, a partir de la inserción de las TIC en el escenario educativo, el programa de investigación, bajo el liderazgo del Grupo Educación e Innovación Educativa, se propuso construir una línea base del nivel de desarrollo de las competencias básicas y ciudadanas, alcanzado por los estudiantes de los grados $6^{\circ}$ y $7^{\circ}$ de las Escuelas Innovadoras del Caribe Colombiano, a partir del desarrollo de estrategias de enseñanza y aprendizaje, mediadas por TIC. Para ello se caracterizó el estado inicial de desarrollo de competencias a partir del desempeño en las Pruebas Estatales Saber 2013 - Colombia y los resultados académicos del primer semestre de 2014 en las instituciones educativas intervenidas, se valoraron los avances en el desarrollo de competencias a partir del uso de TIC en los procesos de enseñanza y aprendizaje, de acuerdo a los estándares del MEN; de igual forma, se sistematizó el impacto del uso de TIC en la enseñanza y aprendizaje en el desarrollo de competencias y se definieron lineamientos de buenas prácticas educativas en uso de TIC, que aporten al diseño de lineamientos de políticas públicas en educación para el Caribe Colombiano

D. Propuesta de lineamientos para una política pública de buenas prácticas en el uso de TIC en las IE del Caribe Colombiano

Con fundamento en el análisis de los hallazgos y resultados de cada proyecto de investigación, se plantea una propuesta de lineamientos de políticas públicas en uso educativo de TIC, que contribuya al desarrollo de una cultura digital con pertinencia.

En el marco del propósito de construcción de esas buenas prácticas, las escuelas intervenidas, vieron limitadas sus posibilidades de desarrollo tecnológico, al no contar con la infraestructura física, dotación y mobiliario necesario y adecuado, los ambientes físicos favorables para el aprendizaje, y lo más preocupante, los servicios de energía eléctrica y conectividad. Ello lleva a reconocer la necesidad de formulación de una política pública en educación, orientada a la fundamentación y promoción de buenas prácticas en uso de TIC con enfoque de equidad social, tanto para el sector urbano como el rural, sin discriminación de estratos sociales. Se trata de promover una política pública, garante del derecho a la educación, a partir del aprovechamiento del potencial de las TIC para inclusión educativa y acceso a la sociedad del conocimiento.

Enfrentar, en tan poco tiempo, la dolorosa realidad del robo, daño y desuso de los equipos suministrados a dichas escuelas por el proyecto y presenciar la existencia de cementerios tecnológicos en algunas de ellas, convoca al reconocimiento de la imperiosa necesidad de formulación de una política pública en educación con responsabilidad civil con la inversión pública, que a partir de la provisión del recurso humano, insumos y servicios de aseguramiento, mantenimiento y soporte técnico permanente a las dotaciones recibidas, garantice la continuidad en el goce de los servicios, y por ende, del derecho a una educación de calidad.

En las últimas décadas, el gobierno nacional, ha emprendido una agresiva campaña de formación docente en uso de TIC, no obstante, su impacto en la calidad educativa de las regiones sobre todo en el Caribe no ha sido significativo. Este constituye un fuerte argumento para la formulación de una política pública de formación docente monitoreada permanentemente en su implementación, tanto por los directivos de las instituciones educativas, como por las autoridades educativas del contexto local, regional y nacional; una política que garantice un seguimiento y evaluación permanente y un mejoramiento continuo; una política que trascienda las estadísticas, hacia la función pedagógica de la escuela de formación de seres humanos. 
La llegada del programa "ICT Capability Building Construyendo capacidad en uso de TIC”, del MEN a las Escuelas Innovadoras, no contó con la acogida esperada; algunos docentes vieron esta iniciativa como una más, como un invento nuevo, como un recargo de trabajo. Ante ello, se hace necesario la formulación de una política pública orientada hacia una gestión educativa que a partir del reconocimiento de las TIC como mediadoras y posibilitadoras del aprendizaje, asuma el área de informática y las salas de informática con una concepción interdisciplinaria; como apoyo al aprendizaje de las diferentes disciplinas y proyectos pedagógicos, superando la concepción asignaturista, del aprendizaje de la máquina y su manejo, como un fin en sí mismo. En este sentido, la política educativa en uso de TIC, debe orientarse al requerimiento de una educación que trascienda las prácticas pedagógicas transmisionistas, instruccionistas y conductistas, que muy poco contribuyen, a la apropiación social de la ciencia, a la generación de conocimiento científico y mucho menos, a la formación de sujetos sociales, activos de derechos; sujetos autónomos, libres y responsables de sus actos, asumiendo así su compromiso con la educación para la equidad social y la construcción de justicia y paz.

\section{CONCLUSIONES}

Durante los años 2014 a 2016, la alianza CIER Norte deja, en la región Caribe Colombiana, capacidades humanas altamente cualificadas y todo un know-how para el futuro desarrollo del capital humano que requiere la región y el país.

La construcción de estos lineamientos de política pública se orientó a propiciar el mejoramiento de las prácticas educativas más allá de lo regional y nacional, para ir avanzando hacia la consolidación de las escuelas oficiales (públicas), a partir de la inversión en infraestructura tecnológica, formación de docentes, la creación y disposición de contenidos educativos digitales en Portal Educativos y el desarrollo de la capacidad investigativa.

Como esta investigación fue respaldada por el MEN y Colciencias, se espera que sea insumo para el Plan Decenal de Educación de Colombia 2016-2026, que está en proceso de construcción y también que sea sujeto de discusión y/o acogida en escenarios académicos por fuera del país ya que al revisar referentes internacionales, se encuentran problemáticas comunes que bien pueden ser abordadas acogiendo o adaptando estas buenas prácticas en uso de TIC y movilizar estas iniciativas de lineamientos para adecuar la legislación pública estatal a las realidades de las escuelas públicas.

\section{REFERENCIAS}

Comisión Europea. Eurydice. (2011) Cifras clave sobre el uso de las tic para el aprendizaje y la innovación en los centros escolares de Europa. Recuperado de: http://eacea.ec.europa.eu/education/eurydice

Departamento Nacional de Planeación DNP. CONPES 3507. (2008). Bogotá, Colombia. Recuperado de: https://sites.google.com/site/ueictcapability/documentosreferentes

Departamento Nacional de Planeación DNP. CONPES 3582. (2009). Bogotá, Colombia. Recuperado de: http://www.colciencias.gov.co/sites/default/files/upload/r eglamentacion/conpes-3582-2009.pdf

Departamento Nacional de Planeación DNP. CONPES 3768. (2013). Bogotá, Colombia. Recuperado de: https://colaboracion.dnp.gov.co/CDT/Conpes/Econ\%C3 \%B3micos/3768.pdf

Dye, Thomas R. (1995). Understading public policy. New Jersey, Estados Unidos: Prentice Hall, Englewood Cliffs.

EACEA Agencia Ejecutiva en el ámbito Educativo, Audiovisual y Cultural. (2011) Uso de las TIC para el aprendizaje y la innovación en los centros escolares de Europa. Unión Europea. Recuperado de: http://eacea.ec.europa.eu/education/eurydice/documents/ key_data_series/129ES.pdf

Gewerc, A. \& Montero, L (2011). Do innovation projects with ICT enhance learning? Experiences from case studies in Galician schools. Journal for Educational Research. Recuperado de: http://www.j-e-r o.com/index.php/jero/article/view/95/106

González, Gilberto et al. (2016) Lineamientos de diseño, implementación y evaluación de estrategias pedagógicas innovadoras mediada por el uso de las TIC. Barranquilla, Colombia. Recuperado http://www.itsa.edu.co/itsacier/cartillaitsacier.pdf

Internet Society (2015). Internet Society global internet report 2015: Mobile evolution and development of internet. Recuperado http://www.internetsociety.org/globalinternetreport/2015 /assets/download/IS_web.pdf

Mestre G. y Díaz D. (2012). Guía para la formación de docentes en la apropiación pedagógica de las TIC. Cartagena, Colombia.

Mestre, G. Alba Cárdenas, Ruby Rojas \& Elsa Ruiz. (2016). Procesos de Formación Docente para el uso de las TIC. En Universidad Tecnológica de Bolívar (Ed.), Buenas Prácticas en uso de TIC en las Escuelas del Caribe Colombiano (pp 129-165). Cartagena de Indias, Colombia: Editorial Universidad Tecnológica de Bolívar.

Pacheco, Alix et al. (2016) Buenas Practicas en Uso de TIC. Cartagena de Indias, Colombia: Editorial Universidad Tecnológica de Bolívar.

Pombo, A. et al. (2016). Lineamientos para la Gestión y Uso de Contenidos Educativos Digitales en el desarrollo de competencias básicas y ciudadanas. Cartagena, Colombia. Recuperado de: http://aprende.colombiaaprende.edu.co/ckfinder/userfiles /files/cartilla02-2.pdf

Vaillant., D. (2013). Programa. TIC y Educación Básica Integración de TIC en los sistemas de formación docente inicial y continua para la Educación Básica en América Latina. Buenos Aires, Argentina UNICEF. Recuperado de:

http://www.unicef.org/argentina/spanish/educacion_Inte gracion_TIC_sistemas_formacion_docente.pdf 